\title{
Hypotension Prediction Index software for management of hypotension during moderate- to high-risk noncardiac surgery: protocol for a randomized trial
}

Kamal Maheshwari ${ }^{1,2^{*}}$, Tetsuya Shimada ${ }^{1,3}$, Jonathan Fang ${ }^{1}$, Ilker Ince ${ }^{1,4}$, Edward J. Mascha ${ }^{5}$, Alparslan Turan ${ }^{1,2}$, Andrea Kurz ${ }^{1,2}$ and Daniel I. Sessler ${ }^{1}$

\begin{abstract}
Background: Hypotension is associated with serious complications, including myocardial infarction, acute kidney injury, and mortality. Consequently, predicting and preventing hypotension may improve outcomes. We will therefore determine if use of a novel hypotension prediction tool reduces the duration and severity of hypotension in patients having non-cardiac surgery.

Methods/design: We will conduct a two-center, pragmatic, randomized controlled trial $(N=213)$ in noncardiac surgical patients $>45$ years old who require intra-arterial blood pressure monitoring. All participating patients will be connected to a Flortrac IQ sensor and EV1000 platform (Edwards Lifesciences, Irvine). They will be randomly assigned to blinded or unblinded arms. The Hypotension Prediction Index (HPI) and advanced hemodynamic information will be universally recorded, but will only be available to clinicians when patients are assigned to unblinded monitoring. The primary outcome will be the effect of HPI software guidance on intraoperative timeweighted average mean arterial pressure under a threshold of $65 \mathrm{mmHg}$, which will be assessed with a WilcoxonMann-Whitney 2-sample, two-tailed test.
\end{abstract}

Discussion: Our trial will determine whether the Hypotension Prediction Index and associated hemodynamic information substantively reduces hypotension during non-cardiac surgery.

Trial registration: ClinicalTrials.gov, NCT03610165. Registered on 1 August 2018.

\section{Background}

Intraoperative hypotension is surprisingly common and, depending upon the definition, has an incidence ranging from 5 to $99 \%$ [1]. Most patients having non-cardiac surgery experience at least one episode during which mean arterial pressure (MAP) decreases to $<65 \mathrm{mmHg}$, often shortly after anesthetic induction [2, 3]. Intraoperative hypotension is associated with complications, including myocardial infarction, acute kidney injury, and mortality [4-7]. A recent randomized controlled trial reported

\footnotetext{
*Correspondence: maheshk@ccf.org; http://www.or.org/

'Department of Outcomes Research, Anesthesiology Institute, Cleveland Clinic, 9500 Euclid Avenue, E-31, Cleveland, OH 44195, USA

${ }^{2}$ Department of General Anesthesiology, Anesthesiology Institute, Cleveland

Clinic, 9500 Euclid Avenue, E-31, Cleveland, OH 44195, USA

Full list of author information is available at the end of the article
}

that preventing intraoperative hypotension reduces the risk of postoperative organ dysfunction by about a quarter. This important result suggests that the association between hypotension and organ injury is at least partially causal and, therefore, amenable to intervention [8].

It seems likely that reducing the frequency, depth, and duration of intraoperative hypotension will reduce organ injury. Currently, anesthesia clinicians respond to blood pressure trends and treat hypotension as necessary when it occurs. But in practice, it is difficult to predict, and therefore prevent, intraoperative hypotension.

The Hypotension Prediction Index (HPI) is derived from arterial waveforms and predicts hypotension, and the software also provides advanced hemodynamic data including cardiac output, vascular elastance, and stroke

(c) The Author(s). 2019 Open Access This article is distributed under the terms of the Creative Commons Attribution 4.0 International License (http://creativecommons.org/licenses/by/4.0/), which permits unrestricted use, distribution, and 
volume-which presumably help clinicians select optimal treatments. HPI uses arterial waveform features, including waveform time, amplitude, area, segment slopes, and complexity features, to predict hypotension, defined by MAP $<65 \mathrm{mmHg}$ for at least one minute [9]. The sensitivity and specificity of HPI for predicting hypotension $5 \mathrm{~min}$ beforehand is $92 \%$ and $92 \%$; it is $89 \%$ and $90 \% 10 \mathrm{~min}$ beforehand and $88 \%$ and $87 \% 15 \mathrm{~min}$ beforehand [9]. HPI software is commercially available in Europe and the United States.

We propose to compare the amount of intraoperative hypotension below a MAP of $65 \mathrm{mmHg}$ in patients requiring invasive arterial pressure monitoring who are randomized to blinded vs unblinded HPI monitoring. Specifically, we will test the primary hypothesis that adding HPI software guidance to the information provided by the invasive arterial pressure monitoring during moderate-to-high-risk noncardiac surgery reduces time-weighted average (TWA) intraoperative hypotension below a threshold of 65 $\mathrm{mmHg}$. Secondarily, we will study the effect of HPI software guidance on TWA MAP below 60 and $55 \mathrm{mmHg}$.

\section{Methods/design}

We will conduct a trial comparing the amount of hypotension in patients requiring invasive arterial pressure monitoring and randomized to blinded vs unblinded HPI monitoring (Fig. 1). We followed SPIRIT recommendations for interventional trial (Addtional file 1). Informed consent will be obtained from all participating patients. The study sites will be the Cleveland Clinic Main Campus and the Cleveland Clinic Fairview Hospital. Ethics committee approval was obtained from the Cleveland Clinic
Institutional Review Board. The trial was registered at ClinicalTrials.gov on July 13, 2018 (NCT03610165) under the principal investigator Kamal Maheshwari.

Written informed consent will be obtained from all included patients. Enrolled patients will be at least 45 years old, have an American Society of Anesthesiologists (ASA) physical status III-IV, be scheduled for elective major non-cardiac surgery with general anesthesia, be planned to have intra-arterial pressure monitoring, and have at least overnight hospitalization. Patients will be excluded if they are pregnant or have known clinically important intra-cardiac shunts, aortic stenosis with valve area $\leq 1.5$ $\mathrm{cm}^{2}$, moderate to severe aortic and/or mitral regurgitation, and moderate to severe mitral stenosis. Patients will also be excluded if they have persistent atrial fibrillation, or congestive heart failure with ejection fraction $<35 \%$. And finally, we will exclude patients scheduled for neurosurgery or cardiovascular procedures.

Potentially qualifying patients will be evaluated during their preoperative anesthesia clinic visits. Written informed consent will be obtained from each, and each will be informed that they may decline to participate or withdraw from the study at any time.

\section{Protocol}

There will be no restriction on type of general anesthesia, and regional anesthesia/analgesia will be permitted. In addition to the routine anesthetic monitoring, an arterial catheter will be inserted for direct pressure monitoring. The catheter will be connected to a FloTrac IQ sensor, and the sensor connected to an EV1000

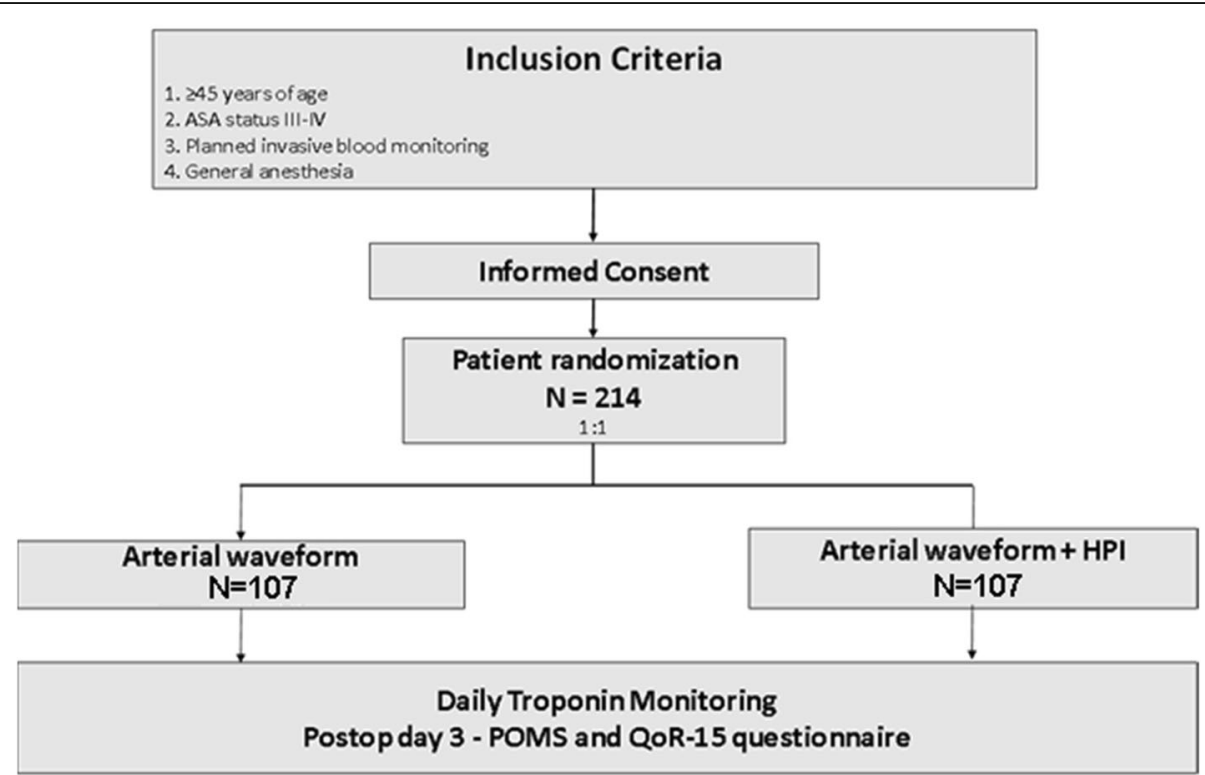

Fig. 1 Study flow chart. ASA American Society of Anesthesiologists, HPI hypotension prediction index, POMS postoperative morbidity survey, QoR15 quality of recovery 
platform which includes HPI software (Edwards Lifesciences, Irvine, CA; Fig. 2).

Shortly before case start, patients will be randomly assigned to blinded or unblinded HPI software monitoring by the investigator not involved in clinical care. Randomization will be stratified by study site, with randomly sized blocks achieving a 1:1 balance within site. Randomization codes will be reproducible, generated using the PROC procedure in SAS statistical software, and implemented via a web-based system (REDCap secure web application). Allocation will thus be concealed, and patients will not be informed of their group assignments. The randomized groups will be:

Blinded HPI software: Arterial waveform and pressures displayed on GE CARESCAPE patient monitor.

Unblinded HPI software: Arterial waveform and pressures displayed on GE CARESCAPE patient monitor and the Hypotension Prediction Index software-enabled EV1000 platform.

Anesthetic dose, the amount and timing of intravenous fluids and use of vasopressor and inotropic drugs will be determined by the anesthesia team and based on available information in both groups. For patients assigned to blinded monitoring, decisions will be based on patient and surgical characteristics, signals from all routine anesthesia monitors, and the arterial waveform.

In patients assigned to unblinded monitoring, clinicians will additionally have access to HPI index, ranging from 0 to 100, corresponding to the likelihood of hypotension in the coming minutes. The system generates an alert to clinicians when HPI reaches or exceeds 85. To standardize interpretation of hemodynamic values, participating clinicians will agree to the conceptual framework presented in Fig. 3, which considers the possibilities of hypovolemia, vasoplegia, and decreased contractility, and recommend potential interventions. To the extent possible, an in-room investigator will assign the HPI alerts to one of the six potential intervention categories: fluids only, fluids and inotropes, fluids and vasopressor(s), vasopressor(s) only, inotropes only, and observation. The investigator's conclusion will be shared with clinicians. Clinicians will nonetheless be free to choose any intervention they believe to be appropriate, and the actual intervention will be recorded.

In all cases, good clinical judgment will prevail and the attending anesthesiologist will take all necessary steps to provide optimal and safe care. Blood and blood products will be given per clinical judgment.

Patients will be blinded to group allocation. Anesthesiologists, surgeons, and other intraoperative personnel cannot be blinded to monitoring allocation. However,

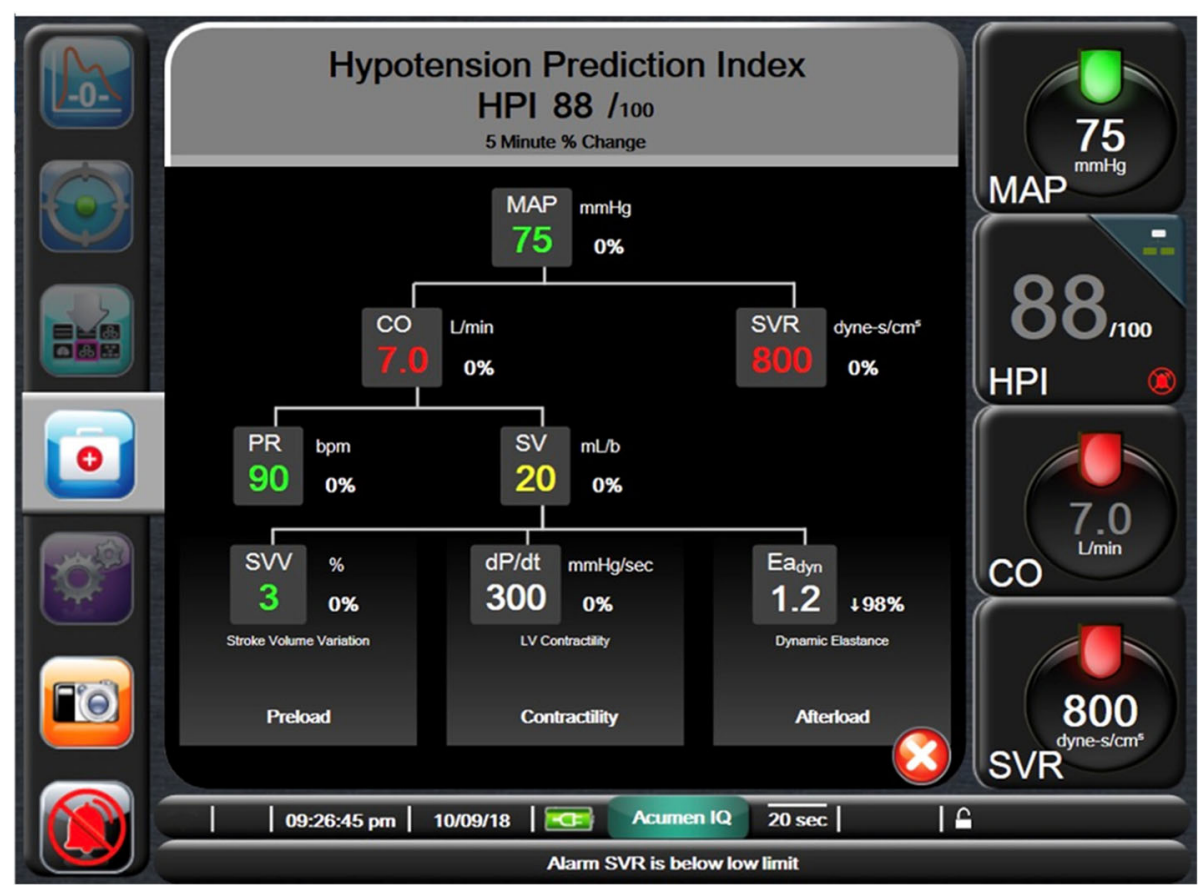

Fig. 2 Advanced hemodynamic information. In the screenshot the Hypotension Prediction Index (HPI) is $88 \%$ and hemodynamic parameters mean arterial pressure (MAP), pulse rate (PR), cardiac output (CO), stroke volume (SV), stroke volume variation (SW), rate of contractility (dP/dt), dynamic elastance $\left(E a_{d y n}\right)$ are shown 


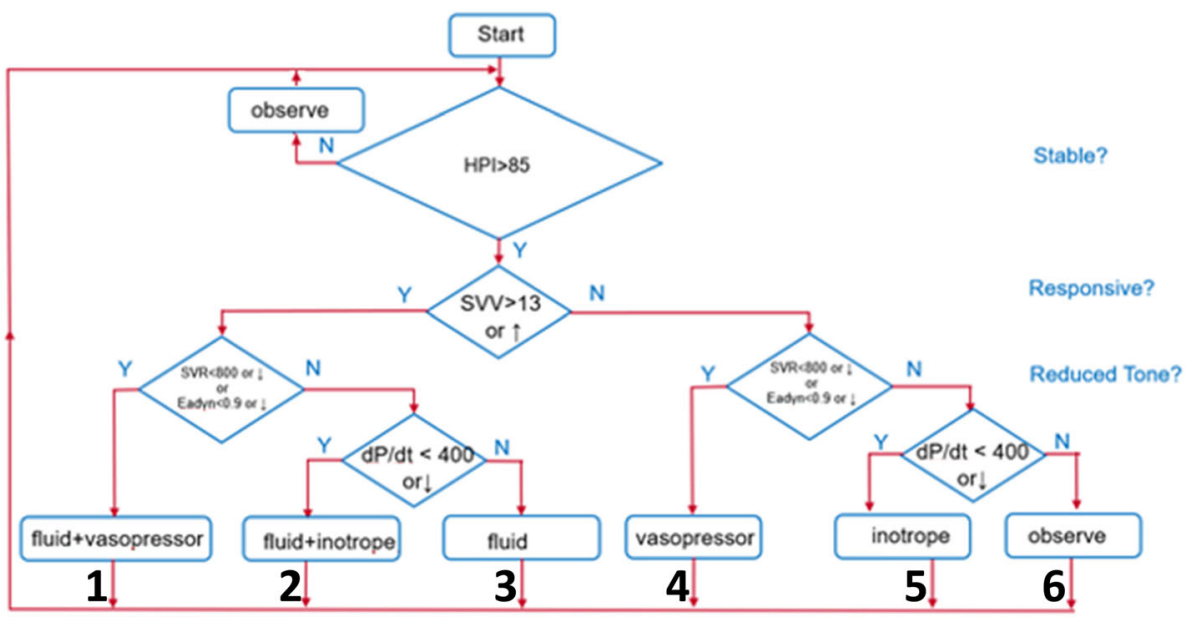

Fig. 3 Conceptual framework for the hemodynamic management. HPI hypotension prediction index, SW stroke volume variation, SVR systemic vascular resistance, $E a_{d y n}$ dynamic arterial elastance

other investigators and the data-analysis team will remain blinded.

Trial-related management will end when surgery finishes. Patient disposition to a critical care unit or routine surgical ward will be determined by the clinical team per routine.

\section{Measurements \\ Baseline information}

We will record demographic and morphometric characteristics, including height, weight, age, sex, ASA physical status, and self-declared ethnicity. As available, we will collect social history (tobacco and alcohol use), medical history (pulmonary disease, cardiovascular disease, neurologic disease, drug use (including statins, $ß$ blockers, oral hypoglycemic agents, and/or insulin), non-steroidal anti-inflammatory drugs (NSAIDs), diabetes (whether insulin-dependent or not), and glucose-tolerance test results, preoperative hemoglobin and hematocrit, blood urea nitrogen and creatinine, electrolytes, preoperative electrocardiogram, and hemoglobin $\mathrm{A}_{1} \mathrm{c}\left(\mathrm{HbA}_{1} \mathrm{c}\right)$.

\section{Perioperative data}

Intraoperative care data will be accessed from the electronic anesthesia information management system. Anesthetic data will include the volatile anesthetic dose in minimum alveolar concentration-hours (MAC-hours), as well as total doses of propofol, opioids, and sedative hypnotics. Hemodynamic and respiratory values, Bispectral Index, and esophageal temperature will be recorded at regular intervals intraoperatively. Blood loss will be estimated; urine output and fluid administration, including colloids and allergenic blood, will be recorded. Intraoperative use and total dose of vasoactive drugs as well as antibiotic administration will be recorded. If available, arterial blood gas results will be recorded.

\section{Blood pressure measurements}

Hemodynamic data from the HPI software will be secured on Cleveland Clinic computers for subsequent analysis and manually recorded at 5-15-min intervals. In the unblinded group an investigator will evaluate HPI alerts and document the probable cause based on advanced hemodynamic parameters using the conceptual framework presented in Fig. 3 and the actual intervention.

\section{Follow-up}

Patients will be followed for the major and minor complications shown in Table 1. Blood will be sampled for generation-4 troponin $\mathrm{T}$ preoperatively, and on the first and second postoperative days while patients remain hospitalized, which will detect $93 \%$ of 30 -day myocardial injury [10]. When troponin $\mathrm{T}$ elevations are detected, patients will be queried about ischemic symptoms and, when practical, an electrocardiogram and echocardiogram will be obtained. Other study outcomes will be appropriately evaluated and documented. Length of stay will be calculated from the end of surgery to discharge or death (Table 2).

\section{Data analysis}

Randomized groups will be compared on baseline variables using the standardized difference (difference in means or proportions divided by the pooled standard deviation), and such variables will be considered balanced if the absolute standardized difference is $<1.96$ sqrt $(2 / N)$, where $N$ is the per group sample size. Imbalanced variables will be adjusted for in all analyses. 
Table 1 Outcomes and measurements

\begin{tabular}{|c|c|c|}
\hline & Outcomes & Measurements \\
\hline 1. Primary & TWA MAP drop under $65 \mathrm{mmHg}$ threshold & Intraoperative record from EV1000 monitor, Edwards Lifesciences \\
\hline \multirow[t]{2}{*}{ 2. Secondary } & TWA MAP drop under $60 \mathrm{mmHg}$ threshold & Intraoperative record from EV1000 monitor, Edwards Lifesciences \\
\hline & TWA MAP drop under $55 \mathrm{mmHg}$ threshold & Intraoperative record from EV1000 monitor, Edwards Lifesciences \\
\hline \multirow[t]{10}{*}{ 3. Exploratory } & Composite of death, stroke, or MINS & $\begin{array}{l}\text { From Cleveland Clinic Perioperative Health Documentation } \\
\text { System PHDS and troponin first three postoperative mornings } \\
\text { while in hospital from EHR }\end{array}$ \\
\hline & Acute kidney injury & AKIN, creatinine \\
\hline & Quality of recovery (QoR-15) & POD 3, patient interview \\
\hline & Post-operative morbidity survey (POMS) & POD 3, EHR \\
\hline & Transfusion requirements (ml) & From PHDS \\
\hline & Amounts of intraoperative crystalloid and colloid (ml) & From PHDS \\
\hline & $\begin{array}{l}\text { Amount of vasoactive: phenylephrine, ephedrine, } \\
\text { nor-epinephrine, epinephrine, dobutamine (mg) }\end{array}$ & From PHDS \\
\hline & Advanced hemodynamic variables & From EV100 \\
\hline & Hospital length of stay & From PHDS \\
\hline & Hospital readmission within 30 days & From EHR \\
\hline
\end{tabular}

AKIN Acute Kidney Injury Network, EHR electronic health record, MAP mean arterial pressure, MINS myocardial injury in non-cardiac surgery, PHDS Perioperative health documentation system, PHDS POD post-operative day, TWA time-weighted average

The treatment effect of HPI guidance (versus no HPI guidance) on the primary outcome of intraoperative time-weighted average (TWA) MAP under a threshold of $65 \mathrm{mmHg}$ will be assessed with a Wilcoxon-Mann-Whitney 2-sample two-tailed test, or analogous test to account for imbalanced baseline variables or non-normal distribution of the outcome, as needed. Secondary and exploratory continuous outcomes will be similarly analyzed. We will assess agreement between clinician and decision support flow chart (ordered decisions from 1 to 6; Fig. 3) using weighted Cohen's kappa, and specifically the Cicchetti-Allison weights [11]. The weighted kappa will accommodate the ordered nature of the rating outcome, giving a weight of 1.0 for perfect agreement, and increasingly smaller weight for larger disagreements.
Randomized groups will be compared on binary outcomes using a Pearson chi-square test or logistic regression to adjust for baseline variables, as needed, and on other outcomes using either the Wilcoxon-Mann-Whitney test or a proportional odds logistic regression model, as appropriate.

For all analyses, focus will be on the estimated treatment effects and their confidence intervals, as well as the $P$ values. SAS statistical software, Carey, NC, will be used for all analyses.

\section{Sample size considerations}

Using our institutional database, we observed a mean (standard deviation) area under a MAP $<65$ of 80 (127) mmHg.min and median [quartiles] of 24 [1, 121]

Table 2 Schedule of study events

\begin{tabular}{|c|c|c|c|c|}
\hline Data & Screening evaluation & Enrollment & Hemodynamic monitoring & Postoperative day 3 \\
\hline Informed consent & $x$ & & & \\
\hline Inclusion/exclusion criteria evaluation & $x$ & & & \\
\hline Medical history & & $x$ & & \\
\hline Demographic & & $x$ & & \\
\hline Vital signs & & $x$ & $x$ & \\
\hline Surgery information & & $x$ & & \\
\hline Monitoring duration & & & $x$ & \\
\hline Device memory data & & & $x$ & \\
\hline Adverse events & & & $x$ & $x$ \\
\hline Hemodynamic management information & & & $x$ & \\
\hline Intraoperative medications & & & $x$ & \\
\hline Quality of recovery & & & & $x$ \\
\hline
\end{tabular}


mmHg.min in a representative sample of patients having non-cardiac surgery (unpublished data). AUC-MAP represents depth and duration of hypotension. AUC-MAP = TWA-MAP $\times$ duration of observation. The data were highly skewed, with a non-trivial proportion of zero values. We therefore used a non-parametric test Wilcoxon-Mann-Whitney test to estimate sample size. Based on these data, a sample size of 213 will provide $80 \%$ power for detecting an approximately $20 \%$ relative reduction in the mean of the primary outcome of AUC-MAP $<65$ mmHg.min.

\section{Discussion}

Our goal is to determine whether using HPI software reduces the duration and severity of intraoperative hypotension. The goal is important because intraoperative hypotension is associated with adverse outcomes, including myocardial injury [12], acute kidney injury [6, 12], and 30 -day mortality $[5,13]$. Furthermore, limited randomized data suggest that hypotension avoidance reduces the risk of postoperative organ failure [8]. To the extent that HPI software reduces hypotension, it may also reduce serious complications.

We will use Flotrac IQ sensors for invasive arterial monitoring because it is necessary for proper functioning of HPI software. In blinded group, we chose not to provide advanced hemodynamic information such as cardiac output because these values are not currently available to our clinicians. Furthermore, HPI is designed to work as a complete hypotension management software and therefore this trial will evaluate both the HPI prediction and the advanced hemodynamic parameters.

HPI and Flotrac IQ are designed to work in conjunction with an arterial catheter. Planned use of an arterial catheter is thus an inclusion criterion, and will restrict enrollment to relatively sick patients having major procedures-but these are also the patients who have the greatest cardiovascular risk. HPI requires a good-quality arterial line waveform. To the extent that the waveform is damped or varies consequent to changes in patient position or other mechanical issues, predictions may be compromised. Our research team will continuously monitor the quality of the arterial waveform and adjust the catheter and system as necessary.

Once hypotension is predicted by the HPI, clinicians will vary in how proactively they respond, and some may poorly comply with our intervention guidelines, which are recommendations rather than requirements. We will allow considerable flexibility on the theory that clinicians will optimize patient safety even though allowing flexibility will add variability to the results. We will, however, record divergences between the algorithm and actual clinical management and plan sensitivity analyses to evaluate the effects of

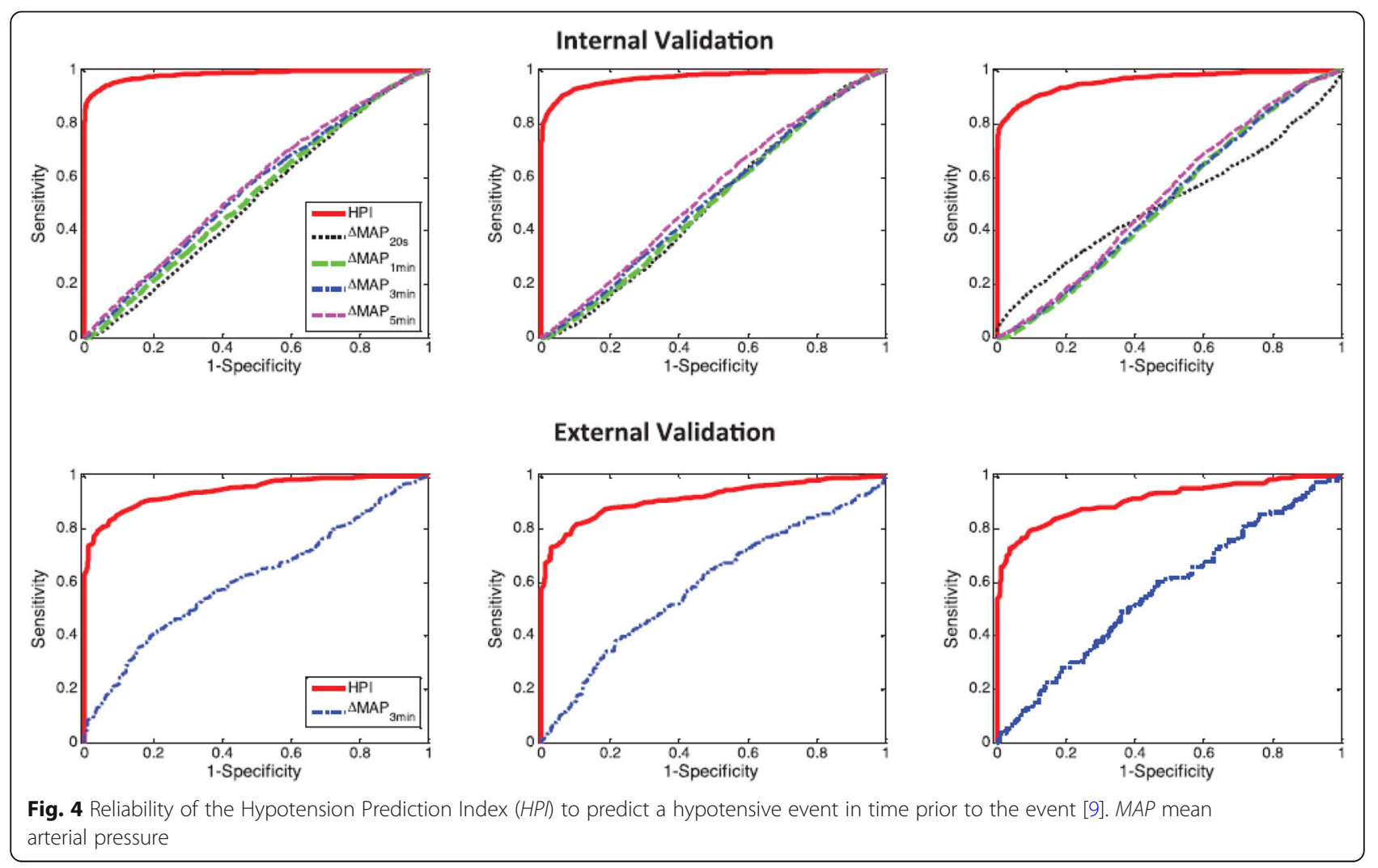


concordant and discordant responses on the extent and duration of hypotension.

HPI software provides reliable prediction of hypotension with good internal and external validation (Fig. 4) [9]. As with many clinical devices, however, HPI software is based on proprietary algorithms and clinicians maybe reluctant to adopt a system that they do not fully understand.

\section{Summary}

We plan a randomized trial to determine whether guiding intraoperative hemodynamic management with HPI software reduces the duration and severity of hypotension during non-cardiac surgery. Since hypotension is associated with perioperative complications, patients may benefit if the HPI and associated hemodynamic data reduce the amount of intraoperative hypotension.

\section{Trial status}

Protocol version 1.0; April 2018. Recruitment began July 12, 2018 and will be completed July, 2020.

\section{Additional file}

Additional file 1: SPIRIT 2013 checklist: Recommended items to address in a clinical trial protocol and related documents. (DOC $130 \mathrm{~kb}$ )

\section{Abbreviations}

ASA: American Society of Anesthesiologists; HPI: Hypotension Prediction Index; MAP: Mean arterial pressure; SAS: Statistical Analysis System; TWA: Time-weighted average

\section{Funding}

Supported by an Investigator Initiated Study grant from Edwards Lifesciences. One Edwards Way, Irvine, CA 92614, USA (e-mail edwards.com)

\section{Availability of data and materials}

Not applicable.

\section{Authors' contributions}

KM, EJM, DIS: concept and study design. KM, TS, JF, II, EJM, DIS: first draft of manuscript and data collection. KM, TS, JF, II, EJM, AT, AK, DIS: editing and critical review. All authors read and approved the final manuscript.

\section{Ethics approval and consent to participate}

Ethics committee approval was obtained from the Cleveland Clinic Institutional Review Board (IRB \#18-557) and informed consent will be obtained from all patients who are enrolled to this study.

\section{Consent for publication}

Not applicable.

\section{Competing interests}

Drs. Maheshwari and Sessler are consultants for Edwards Lifesciences and the Department of OUTCOMES RESEARCH has research contracts with Edwards Lifesciences. These financial interests are within permissible limits established by the Cleveland Clinic Conflict of Interest Policy. The other authors declare that they have no competing interests.

\section{Publisher's Note}

Springer Nature remains neutral with regard to jurisdictional claims in published maps and institutional affiliations.

\section{Author details}

'Department of Outcomes Research, Anesthesiology Institute, Cleveland Clinic, 9500 Euclid Avenue, E-31, Cleveland, OH 44195, USA. ²Department of General Anesthesiology, Anesthesiology Institute, Cleveland Clinic, 9500 Euclid Avenue, E-31, Cleveland, OH 44195, USA. ${ }^{3}$ Department of Anesthesiology, National Defense Medical College, Tokorozawa, Saitama, Japan. ${ }^{4}$ Department of Anesthesiology and Reanimation, Ataturk University School of Medicine, Erzurum, Turkey. ${ }^{5}$ Departments of Quantitative Health Sciences and Outcomes Research, Lerner Research Institute and Anesthesiology Institute, Cleveland Clinic, Cleveland, OH, USA.

Received: 7 December 2018 Accepted: 25 March 2019

Published online: 03 May 2019

\section{References}

1. Bijker JB, van Klei WA, Kappen TH, van Wolfswinkel L, Moons KG, Kalkman CJ. Incidence of intraoperative hypotension as a function of the chosen definition: literature definitions applied to a retrospective cohort using automated data collection. Anesthesiology. 2007;107(2):213-20.

2. Maheshwari K, Turan A, Mao G, Yang D, Niazi AK, Agarwal D, Sessler DI, Kurz A. The association of hypotension during non-cardiac surgery, before and after skin incision, with postoperative acute kidney injury: a retrospective cohort analysis. Anaesthesia. 2018;73(10):1223-8.

3. Green RS, Butler MB. Postintubation hypotension in general anesthesia: a retrospective analysis. J Intensive Care Med. 2016;31(10):667-75.

4. Bijker JB, van Klei WA, Vergouwe $Y$, Eleveld DJ, van Wolfswinkel L, Moons KG, Kalkman CJ. Intraoperative hypotension and 1-year mortality after noncardiac surgery. Anesthesiology. 2009;111(6):1217-26.

5. Monk TG, Bronsert MR, Henderson WG, Mangione MP, Sum-Ping ST, Bentt DR, Nguyen JD, Richman JS, Meguid RA, Hammermeister KE. Association between intraoperative hypotension and hypertension and 30-day postoperative mortality in noncardiac surgery. Anesthesiology. 2015;123(2): 307-19.

6. Sun LY, Wijeysundera DN, Tait GA, Beattie WS. Association of intraoperative hypotension with acute kidney injury after elective noncardiac surgery. Anesthesiology. 2015;123(3):515-23.

7. Walsh M, Devereaux PJ, Garg AX, Kurz A, Turan A, Rodseth RN, Cywinski J, Thabane L, Sessler DI. Relationship between intraoperative mean arterial pressure and clinical outcomes after noncardiac surgery: Toward an empirical definition of hypotension. Anesthesiology. 2013;119(3):507-15.

8. Futier E, Lefrant JY, Guinot PG, Godet T, Lorne E, Cuvillon P, Bertran S, Leone M, Pastene B, Piriou V, et al. Effect of individualized vs standard blood pressure management strategies on postoperative organ dysfunction among high-risk patients undergoing major surgery: a randomized clinical trial. JAMA. 2017;318(14):1346-57.

9. Hatib F, Jian Z, Buddi S, Lee C, Settels J, Sibert K, Rinehart J, Cannesson M. Machine-learning algorithm to predict hypotension based on high-fidelity arterial pressure waveform analysis. Anesthesiology. 2018;129:663-74.

10. Writing Committee for the VSI, Devereaux PJ, Biccard BM, Sigamani A, Xavier D, MTV C, Srinathan SK, Walsh M, Abraham V, Pearse R, et al. Association of postoperative high-sensitivity troponin levels with myocardial injury and 30-day mortality among patients undergoing noncardiac surgery. JAMA. 2017;317(16):1642-51.

11. Cicchetti DV, Allison T. A new procedure for assessing reliability of scoring EEG sleep recordings. Am J EEG Technol. 1971;11:101-9.

12. Salmasi V, Maheshwari K, Yang D, Mascha EJ, Singh A, Sessler DI, Kurz A. Relationship between intraoperative hypotension, defined by either reduction from baseline or absolute thresholds, and acute kidney and myocardial injury after noncardiac surgery: A retrospective cohort analysis. Anesthesiology. 2017;126(1):47-65.

13. Mascha EJ, Yang D, Weiss S, Sessler DI. Intraoperative mean arterial pressure variability and 30-day mortality in patients having noncardiac surgery. Anesthesiology. 2015;123(1):79-91. 